\title{
COMBINED RADIO OBSERVATIONS WITH LOFAR AND THE GIANT UKRAINIAN RADIO TELESCOPE
}

\author{
R. Karlsson*, H. O. Rucker*, G. Mann ${ }^{\dagger}$, A. A. Konovalenko ${ }^{\ddagger}$, \\ F. Breitling ${ }^{\dagger}$, V. V. Dorovskyy ${ }^{\ddagger}$, and C. Vocks ${ }^{\dagger}$
}

\begin{abstract}
A procedure to combine radio data from the Low Frequency Array (LOFAR) [ASTRON, 2010] and the Giant Ukrainian Radio Telescope (GURT) [Konovalenko, et al., 2009] is described. LOFAR is being deployed with 36 stations in the Netherlands and 8 international stations in the neighbouring countries where the central 18 stations close to Exloo in the Netherlands form a core. Data from the Low Band Antennas in the frequency range $10-80 \mathrm{MHz}(10-30 \mathrm{MHz}$ with reduced sensitivity) of the LOFAR station in Bornim close to Potsdam, Germany, 1,500 km from GURT will be used. GURT is being built at the site of the UTR-2 radio telescope close to Charkov, Ukraine. It covers the range $10-70 \mathrm{MHz}$ and the final effective area of GURT will be comparable to the LOFAR core. The GURT data will be converted into narrow bands of the same bandwidth and the same centre frequencies as the LOFAR data. Comparisons of the dynamic spectra from the two stations will be made and the data from the stations can be combined to make use of the long baseline. Scientific focus will be on solar physics within the LOFAR Solar physics and space weather key science project, but the long baseline also allows for investigations of radio sources which require a higher angular resolution than the sun.
\end{abstract}

\section{Introduction}

This paper describes the combination of the low frequency radio telescopes LOFAR, with hub in the Netherlands, and GURT in Ukraine. Both telescopes utilizes thousands small simple electric dipole antennas, which allows the beam to be steered in any desired direction. The frequency ranges of the telescopes overlaps within the range 10-70 MHz, although LOFAR operates with reduced sensitivity below $30 \mathrm{MHz}$.

\footnotetext{
* Space Research Institute, Austrian Academy of Sciences, Schmiedlstrasse 6, A-8042 Graz, Austria

† Astrophysikalisches Institut Potsdam, An der Sternwarte 16, 14482 Potsdam, Germany

$\ddagger$ Institute of Radio Astronomy, Ukrainian Academy of Sciences, Chervonopraporna 4, 61002, Kharkiv, Ukraine
} 
Presently, GURT is under construction and can only operate with a fraction of the final number of antennas. Therefore, UTR-2 (Ukrainian T-shaped Radio telescope-2) is planned to be used for the first experiments with identical DSP-Z receiver backends as for GURT. UTR-2 differs mainly from GURT in terms of its frequency range 8-32 MHz and its single polarisation, whereas LOFAR and GURT both have two polarisations.

The science will mainly address solar physics and space weather within the LOFAR Solar physics and space weather key science project (Solar KSP). In addition, the long baseline between LOFAR and GURT will provide much better angular resolution than required to resolve structures on the sun and this makes the combined telescope useful for studying e.g. radio emissions from giant planets.

In Sections 2 and 3, LOFAR and GURT/UTR-2 are describes, followed in Section 4 by a description of combined observations. Section 5 describes the science which will be made possible by the combination of the radio telescopes and Sections 6 and 7 the data formats produced by the telescopes and the conclusions of the paper, respectively.

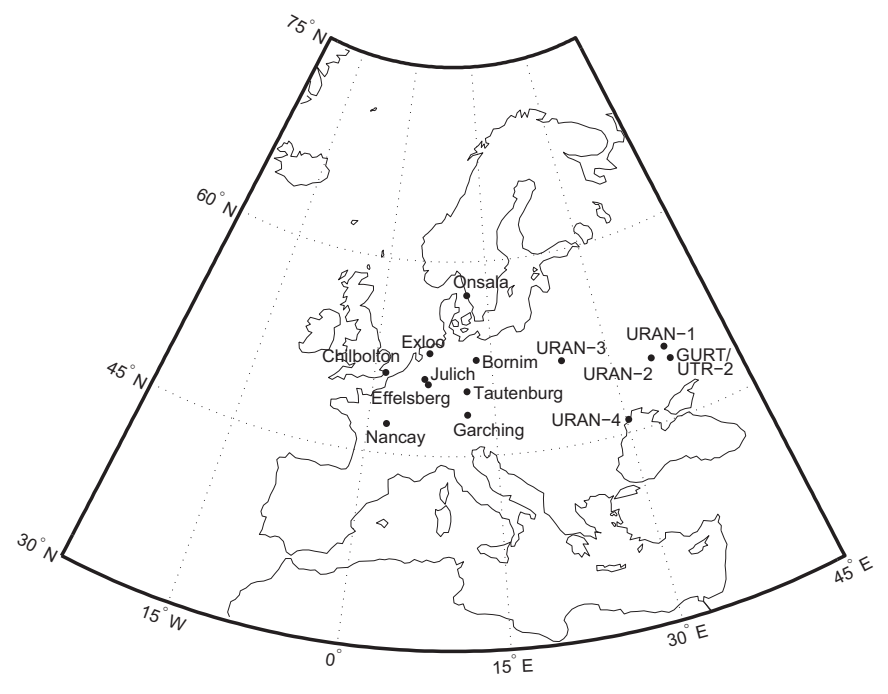

Figure 1: The LOFAR stations and the Ukrainian radio telescopes are shown. In the figure, all the Dutch stations are represented by the core in Exloo (NL), while the international stations in France (Nançay), UK (Chilbolton), Sweden (Onsala), and Germany (Bornim/Potsdam, Tautenburg, Garching, Effelsberg, Jülich) are shown. The Ukrainian radio telescopes GURT and UTR-2 are located at the same site close to Charkov, while URAN-1-4 are situated close to the Ukrainian border to maximize the distance between them. 


\section{LOFAR}

Developed by ASTRON (The Netherlands' Institute for Radio Astronomy), LOFAR is the first of a new generation of radio telescopes and it is being rolled-out with hub in the Netherlands and International stations in the surrounding countries. It has no moving parts and consists of a large number of small inexpensive wire antennas, which allow electronic beam forming. The data from all the stations are sent to a central processing facility in the Netherlands which processes the data on-line.

In total there will be 44 LOFAR stations of which 36 are Dutch stations, divided between 18 core stations close to the hub in Exloo and 18 remote stations spread over the Netherlands. The other eight stations are international stations located in France (Nançay), UK (Chilbolton), Sweden (Onsala), and Germany (Bornim/Potsdam, Tautenburg, Garching, Effelsberg, Jülich).

All LOFAR stations have 96 dual-polarised Low Band Antennas (LBA) for (10-)30-80 $\mathrm{MHz}$. The High Band Antennas (HBA) cover the range 120-240 MHz, which is higher than the frequency of the Ukrainian radio telescopes, and therefore the HBA:s will not be considered any further in this paper. Figure 2 shows a part of a LOFAR LBA field where each antenna comprises two orthogonal dipoles supported by a vertical rod. Further LOFAR specifications and a comparison with GURT are given in Table 1.

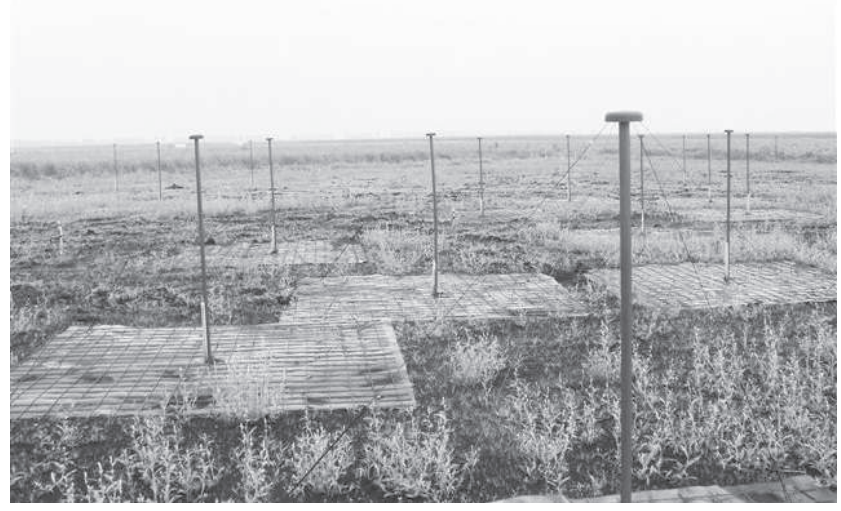

Figure 2: A part of a LOFAR LBA field. The antennas are the thin wires stretching downward from the vertical plastic support rod to the ground plane below. Each station comprises 96 dual-polarised elements. Picture by ASTRON.

\subsection{The LOFAR Super Station}

The LOFAR super station is a French initiative to increase the weight of the their single station by adding approximately 1000 dual-polarised elements separated into 96 small arrays of about 10 elements each and with analog phasing between them. Each of these 
small arrays will be connected to unused inputs on the receiver cards of the LOFAR station. The addition of such a large number of elements will increase the sensitivity for weak sources and the station will essentially form a second LOFAR core. Presently, the antennas are being developed in Nançay and the frequency range may be chosen to extend below $30 \mathrm{MHz}$, which would make the station very interesting to combine with the Ukrainian radio telescopes.

\section{GURT - The Giant Ukrainian Radio Telescope}

The Institute of Radio Astronomy (IRA) of the National Academy of Sciences of Ukraine, Charkov, is responsible for the Ukrainian radio telescopes [Konovalenko et al., 2009]. The telescopes are being upgraded with new DSP-Z backends and UTR-2 is fully operational. GURT is being rolled-out at the site of UTR-2 and presently six of the 100 planned 25element sub-arrays are deployed. In addition, the four smaller URAN radio telescopes for the frequency range 8-32 MHz, have also been equipped with DSP-Z backends. For the first planned campaign, UTR-2 will be used for the band 8-32 MHz, which overlaps partly with the LOFAR band $10-80 \mathrm{MHz}$, but with a lower LOFAR sensitivity in the range 10-30 MHz. GURT, on the other hand, covers the band $10-70 \mathrm{MHz}$ and will, at a more deployed state, be optimal for combined observations with LOFAR.

Specifications for GURT and a comparison with LOFAR are given in Table 1. UTR-2 which has only one polarisation and a maximum effective area of $150,000 \mathrm{~m}^{2}$, has not been included in the table.

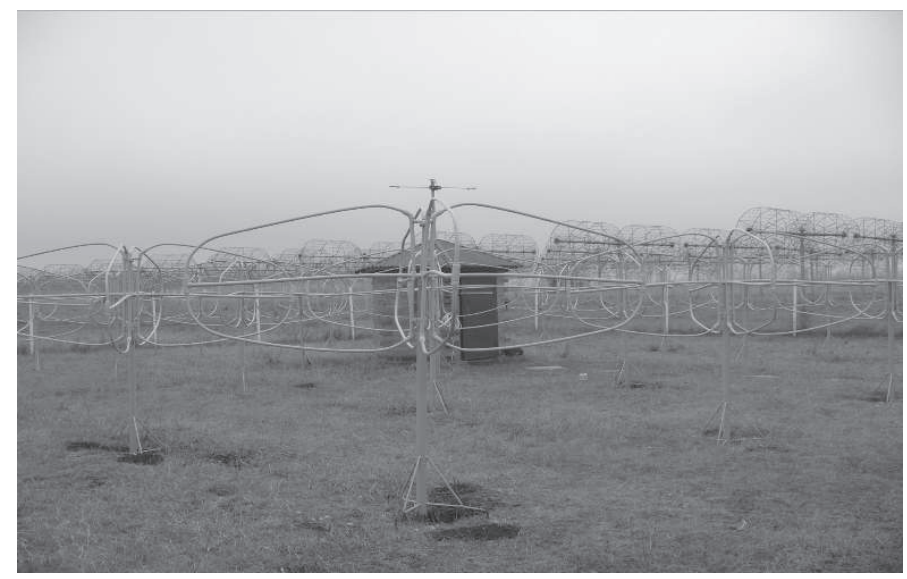

Figure 3: A 25-element GURT dual-polarised sub-array. From Konovalenko et al. [2009]. 
Table 1: Comparison between LOFAR and GURT. The maximum bandwidth given for LOFAR is planned to increase to $48 \mathrm{MHz}$ when new hardware has been installed at the central processing facility.

\begin{tabular}{|l|l|l|}
\hline Parameter & LOFAR & GURT \\
\hline \hline Frequency range & $\begin{array}{l}10-30 \mathrm{MHz}, \text { low sensitivity } \\
30-80 \mathrm{MHz}, \text { Low Band } \\
120-240 \mathrm{MHz} \text {, High Band }\end{array}$ & $10-70 \mathrm{MHz}$ \\
\hline Number of elements & $\begin{array}{l}\text { Total: } 4,224 \mathrm{LBA} \\
\text { Core: } 1,728 \mathrm{LBA}\end{array}$ & $\begin{array}{l}\text { In } 2010: 150 \text { elements } \\
\text { Finally up to } 2,500\end{array}$ \\
\hline Polarizations per antenna & 2 & 2 \\
\hline Max. effective area & $\begin{array}{l}\text { Total: } 350,000 \mathrm{~m}^{2} \\
\text { Core: } 100,000 \mathrm{~m}^{2}\end{array}$ & $>100,000 \mathrm{~m}^{2}$ \\
\hline Max. bandwidth & $32 \mathrm{MHz}(48 \mathrm{MHz})$ & $33 \mathrm{MHz}$ \\
\hline Spectral resolution & $0.76 \mathrm{kHz}$ & $500 \mathrm{~Hz}-7 \mathrm{kHz}$ \\
\hline Temporal resolution & $1.3 \mathrm{~ms}$ & $0.5 \mathrm{~ms}$ \\
\hline Number of freq. channels & $\sim 160 \times 195 / 0.76=42,240$ & 16,000 \\
\hline Dynamic range & $12 \mathrm{bit} \mathrm{ADC}$ & $16 \mathrm{bit} \mathrm{ADC}$ \\
\hline
\end{tabular}

\section{Joint LOFAR and GURT Observations}

The longest LOFAR baseline is about $700 \mathrm{~km}$. By combining LOFAR with GURT the baseline can be extended several times. The distance between GURT and the LOFAR station in Bornim is approximately $1,500 \mathrm{~km}$ and the distance from GURT to the LOFAR core in Exloo is about 2,100 km. The work described in this paper will focus on combining a single LOFAR station, preferably the Bornim station, and GURT or UTR-2. At a later stage, GURT may be combined with the LOFAR core and the LOFAR Super Station.

The joint observations will start with software development followed by joint campaignes of increasing complexity:

1. Development of software to read LOFAR and DSP-Z data formats and to plot dynamic spectra from the stations.

2. Joint campaign with simultaneous LOFAR and GURT/UTR-2 observations of the same object and comparison of dynamic spectra.

3. Simultaneous observations and correlation for VLBI (Very Long Baseline Interferometry). This provides higher angular resolution than needed to resolve structures on the sun.

For VLBI, a number of steps have to be performed. First, the exact positions of the antennas have to be known and search for delays between the antennas will be useful. 
The stations have to operate within the same frequency band and beamform to observe the same part of the sky. Observation data has to be time stamped with a very accurate clock. Data have to be produced in small bands of the same bandwidth and with identical centre frequencies. The data from the DSP-Z receiver will be interpolated to the LOFAR sampling frequency (the opposite is also possible) and the data can be divided into narrow frequency bands identical for both stations. Finally, the data is cleaned and correlated to allow for source size determination.

\section{Science}

The LOFAR science is divided into Key Science Projects (KSP), each led by a particular research group. For the Ukrainian telescopes, all science and observations are handled directly by IRA.

\subsection{The LOFAR Solar Key Science Project}

The LOFAR science is divided into six KSP:s. These are the epoch of reionization, cosmic magnetism of the early universe, deep extragalactic surveys, solar physics and space weather, ultrahigh energy cosmic rays, and transients and pulsars.

The Solar and space weather Key Science Project (Solar KSP) is led by the Astrophysical Institute Potsdam (AIP), Germany, which has deployed a LOFAR station in Bornim close to Potsdam. The European solar community has been invited to take part and contribute to the LOFAR solar science. The Solar KSP utilizes the following three targeted observation modes: radio spectrometry, solar burst imaging, and monitoring of solar activity [AIP, 2009]. In addition, ground-based observations at e.g. the Global H-alpha network (from e.g. Kanzelhöhe in Austria), the solar telescope GREGOR on Teneriffa, and Nançay decametric array, as well as space borne missions such as RHESSI, STEREO, Hinode, SDO, and Solar Orbiter, will give complementing data for LOFAR solar studies.

\subsection{Science from Combined Observations}

The joint LOFAR and GURT observations are restricted to the LOFAR LBA range up to $80 \mathrm{MHz}$ and mainly the Solar KSP will be targeted. The first joint observation with LOFAR and GURT/UTR-2 will take advantage of the solar physics expertise in Potsdam and focus on the sun. It is planned to take place during spring 2011 when the sun reaches a higher declination after the winter. From the first observations, mainly dynamic spectra of the stations will be produced, but further on the data will be combined to make use of the long baseline between LOFAR and GURT. The long baseline will provide an angular resolution much better than required to resolve solar structures and will be useful for observations of e.g. radio emissions from giant planets, studies of Saturn electrostatic discharges [Warwick et al., 1981] and lightning on Uranus [Zarka and Pedersen, 1986], and detection of extrasolar planets by means of their radio emissions. 


\section{Data Formats}

The LOFAR stations and the Ukrainian DSP-Z receiver produce different data formats and as an additional complication, they do not operate at the same sampling frequency. Below, the LOFAR formats relevant for the joint measurements and the DSP-Z format are briefly explained.

\subsection{LOFAR Data Formats}

Descriptions of the LOFAR data formats can be found at http://usg.lofar.org. Among these formats, the dynamic spectrum data, the radio sky image cubes, and the single station imaging data are most relevant for the combined observations.

\subsection{GURT and the DSP-Z Back-end}

The DSP-Z receiver backend has a sampling frequency of $66 \mathrm{MHz}$ and a 16 bit ADC, which provides higher dynamic range than LOFAR. DSP-Z can operate in two modes, the spectrum mode and the waveform mode. The spectrum data requires substantially less space than the waveform data. For combined observations with LOFAR, the spectrum data can be used when dynamic spectra of the stations are compared, but for cases when the LOFAR and DSP-Z data will be correlated, the waveform data is preferred and must be converted to LOFAR format in several step involving e.g. resampling of the data to the LOFAR sampling rate.

\section{Conclusion}

The joint observations with LOFAR and GURT is an important step to enhance the performance of both radio telescopes. For such a combination, the long baseline has the potential to enhance the angular resolution and the increase of effective area will enable more sensitive studies to be performed. A future addition of the LOFAR Super Station will enhance the performance even further. The solar science within the LOFAR Solar KSP will benefit from the joint observations and the enhanced angular resolution and sensitivity will improve the studies of radio emissions from e.g. giant planet magnetospheres, planetary lightning, and extrasolar planets.

Acknowledgements. RK acknowledges the OELZELT stipend of the Austrian Academy of Sciences.

\section{References}

ASTRON, LOFAR Technical Information, 2010. http://www.astron.nl/radioobservatory/technicalinformation/lofar-technical-information 
Astrophysikalisches Institut Potsdam, Key Science Project Solar Physics and Space Weather with LOFAR, 2009. http://www.aip.de/groups/osra/documents/LOFAR_Solar_Sience.pdf

Karlsson, R., H. O. Rucker, G. Mann, F. Breitling, C. Vocks, A. A. Konovalenko, V. V. Dorovskyy, Combination of Radio Observations from LOFAR and the Giant Ukrainian Radio Telescope, Presentation at the Solar Key Science Project Workshop IV, Potsdam, 2010. http://www.aip.de/groups/osra/sksp/SKSP-workshopIV

Konovalenko, A. A., et al. (18 co-authors), Solar radio emission and space weather studies with the ground-based existing (and future) low-frequency radio telescopes, Presentation at the Solar Key Science Project Workshop II, Potsdam, 2009. http://www.aip.de/groups/osra/sksp/SKSP-workshopII

Warwick, J. W., J. B. Pearce, D. R. Evans, T. D. Carr, J. J. Schauble, J. K. Alexander, M. L. Kaiser, M. D. Desch, B. M. Pedersen, A. Lecacheux, G. Daigne, A. Boischot, and C.H. Barrow, Planetary Radio Astronomy observations from Voyager 1 near Saturn, Science, 212, 239-243, 1981.

Zarka, P., and B. M. Pedersen, Radio detection of Uranian lightning by Voyager 2, Nature, 323, 605-608, 1986. 